\title{
Mehrzahl der Haushalte hygienisch bedenklich
}

\author{
Vor allem Kühlschrank und Badezimmerfugen mit Bakterien und Schimmel belastet
}

Wahrscheinlich sind zwischen Joghurt und Salat im Kühlschrank eines Haushaltes mehr Keime als in der Toilette zu finden. Zu diesem Ergebnis kommt eine aktuelle Studie des Hygiene Council, einem internationalen Zusammenschluss von Experten aus dem Bereich Hygiene. Am häufigsten sind demnach Badezimmerfugen, Kühlschrankinnenräume und Küchentücher von krankheitserregenden Keimen befallen. Im Bakterientest scheitern gar 70 Prozent der Haushalte. Der internationale Rat von Hygieneexperten untersuchte ausgewählte Gegenstände in den Haushalten von neun verschiedenen Ländern auf Bakterien und Schimmel. Offenbar ist das Wissen über keimanfällige Haushaltsgegenstände und wie man diese gezielt reinigt, mangelhaft.

„Die Ergebnisse der Studie haben uns gezeigt, dass wir noch einen langen Weg vor uns haben, wenn es darum geht, Keime wirkungsvoll zu bekämpfen“, sagt Prof. John Oxford, Professor der Virologie am St. Bartholomew's \& The Royal London Hospital und Vorsitzender des Hygiene Councils. Insgesamt erwiesen sich 33 Prozent der bakteriellen Proben und 51 Prozent der Proben gegen Schimmel als ungenügend. Es wird offensichtlich noch nicht genug getan, um die Problemzonen im Haushalt wirkungsvoll zu kontrollieren. Den Grund für die Hygienemängel sieht Oxford weniger im Zeitmangel für die Reinigung, sondern vielmehr im fehlenden Wissen über die einzelnen Bakterienund Schimmelherde im Haushalt.

\section{Vom Küchentuch bis zur Computermaus}

Für die Untersuchungen besuchte der Hygiene Council insgesamt 180 Familien in neun verschiedenen Ländern - Großbritannien, USA, Deutschland, Kanada, Südafrika, Saudi-Arabien, Malaysia, Australien und Indien -, die sich bereit erklärten, an der Studie teilzunehmen. Alle Familien wurden im Vorfeld speziell geprüft und ausgewählt, um möglichst alle sozioöko-

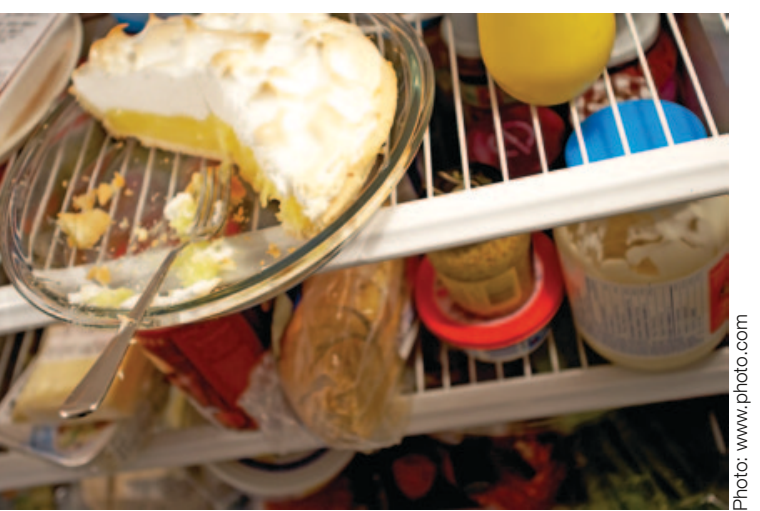

ühlschrankinnenräume zählen zu den am häufigsten kontaminierOrten im Haushalt.

nomischen Hintergründe mit einzubeziehen. In jedem Haushalt wohnten zudem mindestens zwei Kinder unter zehn Jahren, darunter eines unter zwei Jahren. Insgesamt wurden pro Haushalt sechs Objekte untersucht, die bekannt dafür sind, dass sie besonders stark von Bakterien und Schimmel betroffen sind. Dies waren Küchentücher, Haltegriffe von Wasserkochern, Computertastaturen und -Mäuse, Haltegriffe von Kinderwägen, Badezimmerabdichtungen sowie Kühlschrankinnenräume.

Alle Objekte wurden auf Bakterien und Schimmelpilze untersucht: Viermal wurden die Objekte in einem Abstrich einzig nach Bakterien untersucht, in zwei weiteren Abstrichen wurde die Verbreitung von Bakterien und Schimmelpilzen festgestellt. Zusätzlich bewerteten die Experten, wie sauber die jeweiligen Flächen bei bloßer Betrachtung wirken. Damit konnte die scheinbare und die tatsächliche Verschmutzung der Haushaltsobjekte verglichen werden. Die an der Studie beteiligten Familien füllten zudem einen Fragebogen zu ihrer Wahrnehmung und Einstellung der Hygiene im Allgemeinen aus, um ihr Hygieneverhalten einzuschätzen.

\section{Brutstätten für Bakterien und Pilze}

Die Studie identifzierte vor allem Badezimmerfugen als Sammel- und Vermehrungsort für Bakterien und Schimmel. Im Bakterientest erzielten mit Ausnahme der USA und Kanada alle untersuchten Länder ungenügende Ergebnisse. Von den Befragten gaben jedoch insgesamt 52 Prozent an, regelmäßig antibakterielle Reinigungsmittel zu verwenden. Es muss also davon ausgegangen werden, dass diese in vielen Haushalten entweder nicht richtig oder nicht an der richtigen Stellen verwendet werden.

Nach den Badezimmerfugen sind Kühlschrankinnenräume am zweithäufigsten mit Bakterien und Pilzen kontaminiert. Obwohl die meisten auf den ersten Blick sauber erschienen, wurden 46 Prozent aller untersuchten Kühlschränke bakteriell als ungenügend eingestuft, in 44 Prozent aller Kühlschränke wurden gar Schimmelkulturen nachgewiesen. In der Befragung gaben nur 54 Prozent der Teilnehmer an, ihren Kühlschrank einmal monatlich zu reinigen. Eine monatliche Reinigung ist aber sehr wichtig, damit sich Bakterienund Schimmel nicht weiter ausdehnen können. Auch viele Küchentücher bestanden den bakteriellen Test nicht (36\%). Zwar gab knapp die Hälfte der Befragten an, das Küchentuch regelmäßig zu wechseln, doch werden die Tücher häufig bei zu tiefer Temperatur gewaschen, so dass viele Bakterien den Waschgang überstehen. Um Bakterien wirkungsvoll zu beseitigen, ist eine Waschtemperatur von mindestens 60 Grad notwendig. Ein Prozent der Befragten gab gar an, die Tücher nie zu wechseln.

„Die Ergebnisse der Studie zeigen, dass bestimmte Bereiche des Haushalts oftmals vernachlässigt werden, wenn es um eine effiziente Hygienepraxis geht", sagt John Oxford. Haltegriffe von Wasserkochern, Kinderwagen oder Computertastaturen- und -mäuse würden zwar sehr häufig berührt, aber nur sehr selten gereinigt, so dass sich krankheitserregende Mikroorganismen dort ungestört vermehren können. Aber auch bei regelmäßiger Reinigung mit gewöhnlichen Reinigungsmitteln könnten teilweise noch viele Mikroorganismen überleben und später Krankheiten übertragen.

Quelle: Presseaussendung Hygiene Council/ Reckitt Benckiser 